\title{
Beitrag zur Kenntniss der Bindesubstanzen bei
}

\section{Avertebraten.}

\author{
Von \\ Nr. F. Forster, Privatdocent in München.
}

Bei Gelegenheit seiner Studien über die Bindesubstanzen der wirbellosen Thiere ersuchte mich Professor J. Kollmann, einige vergleichende Untersuchungen über das chemische Verhalten gewisser an Bindesubstanzen reichen Organtheile niederer Thiere zu machen und speciell zu prüfen, ob in denselben leimgebende Substanz, wie in dem Bindegewebe der Wirbelthiere, sich vorfinde.

Bekanntlich ist es nicht gerade leicht, wenn nur kleine Mengen der Gewebe zur Verfügung stehen, die Gegenwart von leimgebendem Gewebe resp. Leim, in welchen diese übergeführt werden müssen, nachzuweisen. Nach einigen Voruntersuchungen, die nicht weiter interessiren, habe ich vorerst aus sehr geringen Quantitäten von Geweben höherer Thiere, die sicher leimgebende Substanzen enthalten, Leim zu erhalten versucht und zwar wurde zu dem Zwecke frisches nicht ausgelaugtes Ochsenfleisch mit oder ohne Zusatz von Wasser in eine Glastöhre gebracht, die Röhre zugeschmolzen und sodann kürzere Zeit hindurch bei $130^{\circ} \mathrm{C}$. im Luftbade erhitzt. Die so gewonnene zum Theile gelöste Masse wurde mit heissem Wasser extrahirt, heiss filtrirt, und das Filtrat auf etwa 5-10 Cc. eingedampft. 20 Gramm des frischen Fleisches ohne Wasserzusatz auf solche Weise behandelt gaben eine Flüssigkeit, welche nicht unmittelbar beim Erkalten, jedoch nach etwa 12-24 stündigem Stehen in der Kälte zu einer zitternden Gallerte erstarte. 10-15 Gramm frischen Fleisches mit etwa $10^{\prime \prime}$ Wasser in die Röhre eingeschlossen und gleicherweise behandelt lieferten ebenfalls noch nach dem Erkalten und mehrstündigem Stehen an einem kühlen Orte eine deutlich erkennbare zitternde Gallerte.

Wie das Säugethierfleisch wurden nun auch die mir von Professor Kollmann übergebenen frischen Präparate, welche von fremden histologischen Bestandtheilen möglichst gereinigt und sorgfältig abgewaschen fast nur die Bindesubstanzen der niederen Thiere darstellten in der angeführten Weise, nur unter Zusatz von destillirtem Wasser verwendet. Zur Untersuchung kamen so: 
1. $5 \mathrm{Gramm}$ des bei $100^{\circ}$ getrockneten Mantels von Unio margaritifer und Anodonta cygnea, was mindestens 30-40 Gramm des frischen Organs entspricht.

2. 11 Gramm bei $50^{\circ}$ getrockneter Muskeln von Sepia officinalis, was ungefähr 40-50 Gramm ies frischen Fleisches entspricht.

3. 7.4 Gramm an der Luft getrockneter Haut von Sepia officinalis und Eledone moschata, welche vorher mit kaltem destillirtem Wasser ausgewaschen worden.

4. 1 Gramm des bei $50-60^{\circ}$ getrockneten Kopfknorpels von Cephalopoden.

Bei keiner der untersuchten Proben konnte auch bei starker Einengung des schliesslich erhaltenen Filtrates eine Gallertbildung beobachtet werden, auch nicht nach 24 stündigem Stehen an einem kühlen Orte. Als nun die eingedickten Proben in Porzellanschalen bei einer Temperatur, die $10^{\circ} \mathrm{C}$. kaum überschritt, an der Luft stehen blieben, erwiesen sie sich nach Verlauf von 2-3 Tagen als dicke Masse, welche an der Schale stark klebte. Der darin befindliche Glasstab konnte nur mit knackendem Geräusche und mit Hinterlassung eines kleinen in die Höhe gezogenen spitzen Kegels, der sich allmählig wieder verflachte, aus der Masse ausgezogen werden, während ihm etwas der klebrigen Substanz anhängen blieb.

Ich bemerke, dass diese Erscheinung bei allen Proben, auch bei der geringen Menge der verarbeiteten Cephalopodenkopfknorpel, aus denen Schlossberger ${ }^{1}$ ) nach $y / 4$ stündigem Kochen bei vier Atmosphären-Druck einen chondrinartigen, aber nicht gelatinirenden Körper erhalten, in geringerem oder höherem Maasse auftrat, der Schätzung nach jedoch in dem Extrakte des möglichst rein gewonnenen Muskelfeisches der Sepien am stärksten sich gestaltete.

Bei weiterem Eintrocknen in kühler Luft blieb schliesslich eine harte, etwas glänzende und spröde Masse zurück, die in ihrem Ansehen nur wenig von gewöhnlichem getrocknetem Tischlerleim verschieden schien, sich in wenig kaltem Wasser jedoch allmählig und vollständig löste.

Es konnte vermuthet werden, dass die reichliche Gegenwart von Extractivstoffen etc. eine Gallertbildung verhindert hätten. Um dieselben zu entfernen, habe ich nun den von Nr. 2 gewonnenen Auszug in kaltem Wasser aufgenommen und der Dialyse unterworfen,

1) Schlossberger, Chemie der Gewebe, 1856, S. 13. 
die Probe von Nr. 3 dagegen in wenig Wasser gelöst und mit $90 \%$ igem| Alkohol gefällt, in dem bekanntlich Leim unlöslich ist. Der Inhalt der Dialysenzelle sowohl wie der reichliche in Wasser gelöste Alkoholniederschlag gaben, auf dem Wasserbade zum Syrup eingedampft, auch jetzt keine Gallerte beim Erkalten, sondern erst nach mehrtägigem Stehen an der Luft die gleiche klebrige Masse wie früher.

Ausser den obigen 4 Organen oder Organtheilen wurden noch 13 Gramm der im warmen Luftstrome getrockneten Schulpen der Sepia officinalis in der erwähnten Weise untersucht, allein das eingeengte Filtrat bildete hier eine schmierige Masse, die allmählig eintrocknete, ohne eine klebrige Beschaffenheit anzunehmen. Es ist das das gleiche Resultat, das J. Müller und Schlos sberger ${ }^{1}$ ) bei ähnlicher Behandlung des Os sepiae und von Molluskenschalen erhielten.

Da die Gallertbildung beim Erkalten des eingedickten Saftes, der auf die oben angegebene Weise aus Säugethierfleisch gewonnen wurde, dafür spricht, dass die angewandte Methode der Darstellung von Leim und des Nachweises von leimgebendem Gewebe in thierischen Organen ausreichend ist, so scheint aus dem Gesagten hervorzugehen, dass gew öhnlich er gelatinirender Leim in den durch die genannte Behandlung aus den Organen niederer Thiere erhaltenen Flüssigkeiten nicht oder nur in sehr geringen Mengen zugegen ist.

Das verschiedene Verhalten der frischen Bindesubstanzen der niedern und höhern Thiere gegenüber der gewählten Untersuchungsmethode lässt sich nun meiner Auffassung nach auf zweierlei Weise erklären und zwar müssen wir entweder annehmen, dass die untersuchten Bindesubstanzen der niedern Thiere überhaupt nicht oder nur zum geringsten Theile aus leimgebendem Gewebe bestehen, oder dass die organische Grundlage der genannten Substanzen beim Kochen zwar einen leimartigen Körper liefert, der sich jedoch von den Eigenschaften des aus dem Bindegewebe höherer Thiere gewonnenen Leimes unterscheidet.

Für diese Auffassung dürfte der Umstand sprechen, dass das Muskelfleisch der Sepien, das bekanntlich vielfach als menschliches Nahrungsmittel verzehrt wird, beim Kochen mit Wasser nicht weich

1) A. a. 0. S. 13 , 
wie das Fleisch der pflanzenfressenden Säugethiere, sondern derb, selbst lederartig wird.

Ob aus grössern Mengen des Sepienfleisches doch nachweisbar Leim sich darstellen lässt, dessen Eigenschaften genatter geprüft werden könnten, scheint nach einer Angabe Hoppe-Seyler's ${ }^{1}$ ) nicht unmöglich zu sein.

Mit Sicherheit aber lässt sich die vorliegende hauptsächlich histologisch-interessante Frage vielleicht durch Verdauungsversuche mit Trypsin nach der jüngsten Mittheilung von Ewald und W.

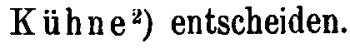

München, am 8. Dezember 1876.

\section{Die Jodreaktion der Knorpel- und Chorda-Zellen.}

Von

Professor W. Neumann in Königsberg i. Pr.

Hierzu Tafel IV.

In meinem Aufsatze „Bemerkungen über das Knorpelgewebe und den Ossifikationsprozess" ${ }^{3}$ ) habe ich es als eine Eigenthümlichkeit der Knorpelzellen hervorgehoben, dass dieselben unter der Einwirkung von Jodlösungen "eine rothbraune oder bei intensiverer Einwirkung schwarzbraune Farbe annehmen. Dieselbe Beobachtung theilt Ranvier in seinem Traité technique d'histologie an mehreren Stellen ${ }^{4}$ ) mit, indem er die Farbe als brun-acajou (Mahagonibraun) bezeichnet, und dieselbe auf die Anwesenheit von Glycogen in den Knorpelzellen bezieht. Da die Thatsache hierdurch ein erhöhtes

1) Tübinger mediz.-chem. Untersuch. S. 586.

2) Verhandlungen des naturhist.-mediz. Vereins zu Heidelberg, I. Bd., 5. Heft. 1876.

3) Archiv d. Heilkunde XI p. 414.1870.

4) Ranvier l. c. p. $273,279,296$. Ob R. bereits früher Angaben über diesen Gegenstand gemacht hat, bin ich aus den Jahresberichten nicht zu ermitteln im Stande gewesen. 